\title{
Proof of an Infinite Number of Primes-Twins
}

\author{
Baibekov S. N. \\ R\&D center "Kazakhstan engineering" \\ Astana c., Kazakhstan
}

Durmagambetov A.A.

Faculty of Mathematics, L.N.Gumilyov Eurasian National University, Astana c., Kazakhstan

\begin{abstract}
This paper is devoted to the theory of prime numbers. In this paper we first introduce the notion of a matrix of prime numbers. Then a number of theorems are proposed in the paper, which together with the theorems of Dirichlet and Euler allow us to prove the infinity of prime twin numbers.
\end{abstract}

Keywords: Prime numbers, prime-twins, composite numbers, integers, algorithm, arithmetical progression, matrix of prime numbers, special factorial.

\section{INTRODUCTION}

It is known that a prime number called natural (positive integer) number (except 1) which is divisible only by 1 and itself. If the natural number (except for one) is not a prime, it is called a composite number.

It is also known that the primes-twins are adjacent prime numbers, the distance between which is equal to 2. A pair of primes-twin, sometimes referred as the usual word "twins". For example, the numbers 11 and 13, 17 and 19 are also twins, but the following adjacent primes 23 and 29 are not twins etc .

There is still a lot of open issues regarding primes. The most famous of them were marked by the 5th International Mathematicians Congress. One of the issues is about proving the infinite number of twins. This problem, which has not been resolved for more than 2,000 years, now named as the second Landau problem.

In 2013, the American mathematician YitangZhang from the University of New Hampshire proved that there are infinitely many pairs of prime numbers separated by a distance greater than 2 , but less than 70 million. Thus, he proved that the number of pairs of prime numbers $\left(p_{i}, p_{i+1}=p_{i}+n\right)$ is infinite, where $2<n \leq 70000000$. Later, James Maynard improved this result to 600. In 2014, a group of scientists under the direction of Terence Tao (Polymath Project), improved this result to 246 [1].

The aim of this work is the proof of an infinite number of primes-twins.

To solve this problem, we have proposed a new method, which allows to evaluate the infinity of primes-twins empirically [2]. Then, in work [3] we proposed a different analytical proof, which gives a correct result of the infinity of the twins. In present work, we aimed to make the proposed evidence infinity twins as possible "elementary" and more pronounced. We believe that the successful use of the fundamental works of world-famous scientists (Eratosthenes, Wilson, Dirichlet, Euler and others) has enabled us to do this. First, for convenience, we introduce the following notation. As it is known sequential multiplication of natural numbers is called factorial: $\prod_{i=1}^{n} i=n$ ! Subsequently, consistent multiplications of prime numbers will be encountered often, so we use the following notation for such cases:

$$
2 * 3 * 5 * 7 * 11 * \ldots * p_{n}=\prod_{i=1}^{n} p_{i}=p_{n} !^{\prime} .
$$


Here $p_{i}$ - a prime number with a serial number $i$. The combination of symbols $p_{n} !^{\prime}$ means extremely consistent multiplication of prime numbers from 2 to $p_{n}$. It will be called a special factorial (or primorial) of prime number $p_{n}$. For example, $p_{4} !^{\prime}-$ a special factorial of fourth prime number $p_{4}=7$ or $p_{4} !^{\prime}=7 !^{\prime}=2 * 3 * 5 * 7=210$.

\section{Matrix of Prime Numbers}

This paper will attempt to prove the infinite number of twins by using the developed matrices of prime numbers,

Representing the set of natural numbers (except number 1) in the form of a matrix family $A_{k}$ with elements of $a\left(k, i_{k}, j_{k}\right)$, where $i_{k}$ - serial number of rows, $j_{k}$ - serial number of columns, $k$ - serial number of matrix $A_{k}$.

$a\left(k, i_{k}, j_{k}\right)=\left(i_{k}+1\right)+p_{k} !^{\prime}\left(j_{k}-1\right)=l_{k}+D_{k}\left(j_{k}-1\right)$,

where $j_{k}=1,2, \ldots, \infty ; i_{k}=1,2, \ldots, p_{k} !^{\prime} . l_{k}=i_{k}+1$ - the first number of plurality of numbers that are in $i_{k}$ row. Note, index $k$ of the parameters $i$ and $j$ denotes affiliation of these parameters to matrix $A_{k}$.

It appears from the equation (1) that the sequence of numbers in any row of the matrix $A_{k}$, is an arithmetic progression with first term which is equal to $l_{k}=i_{k}+1$. The difference in this progression is $D_{k}=p_{k} !^{\prime}$.

Wherein the maximum number of rows of $A_{k}$ matrix must be equal to the special factorial $p_{k}$ !', i.e.

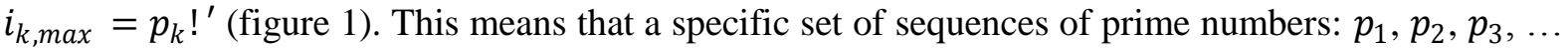
, $p_{k}$ (note, $p_{k}$ is the last prime number, which corresponds to this matrix) has to comply with each matrix with the serial number $k$. The number of columns can be arbitrarily large, up to infinity.

As it is known, one number is not a prime number, otherwise all the numbers that are multiples of the unit would be a composite number. Number 1 is also not a composite number, since it is not divided into different numbers. That is why the number 1 in all matrices separately located in the upper left corner (Figure 1). For clarity reasons only four matrices $A_{1}, A_{2}, A_{3}$ and $A_{4}$ are shown in Figure 1. The number of rows of other matrices exceeds the paper size, so they are not shown in Figure 1.

\section{Lemma 1.}

Any integer $Z>1$ occupies only one specific place in the matrix $A_{k}$. Moreover, the serial numbers $j_{k}$ and $i_{k}$ respectively column and row, in which a given number is, are determined in a unique way:

$$
\begin{gathered}
\boldsymbol{j}_{\boldsymbol{k}}=\text { integer }\left(\frac{Z-2}{p_{k} !^{!}}\right)+\mathbf{1}(2-1) \\
\boldsymbol{i}_{\boldsymbol{k}}=r_{\boldsymbol{k}}+\mathbf{1}(2-2)
\end{gathered}
$$

where $r_{k}$ - residue obtained by dividing the $(Z-2)$ on $p_{k} !^{\prime}$, i.e.:

$(Z-2)=r_{k}\left(\bmod \boldsymbol{p}_{\boldsymbol{k}} !^{\prime}\right)(2-3)$

\section{Proof of lemma 1.}

Imagine $A_{k}$ matrix in the form of a plurality of cells which are arranged in all rows and columns of the given matrix (Figure 1). To each cell, in which only one number can be located, must comply with certain couple "coordinate" ( $i_{k}$ and $\left.j_{k}\right)$.

If assume that the number $Z$ occurs twice in the matrix $A_{k}$. I.e.

$$
Z=a\left(k, i_{k}, j_{k}\right)=a\left(k, n_{k}, m_{k}\right)
$$

where $n_{k}, m_{k}$ - serial numbers of rows and columns, in which the number $Z$ occurs the second time. Then, from equation (1) follows, that

or

$$
\begin{aligned}
& \left(i_{k}+1\right)+p_{k} !^{\prime}\left(j_{k}-1\right)=\left(n_{k}+1\right)+p_{k} !^{\prime}\left(m_{k}-1\right) \\
& i_{k}-n_{k}=p_{k} !^{\prime}\left(m_{k}-j_{k}\right) .
\end{aligned}
$$

Since $i_{k} \leq p_{k} !^{\prime}$ and $n_{k} \leq p_{k} !^{\prime}$, it leads to the deduction that $i_{k}-n_{k}<p_{k} !^{\prime}$. Therefore, the last equality holds only when

$i_{k}=n_{k}$ и $j_{k}=m_{k}$

It follows that any number in any matrix $A_{k}$ is presented in a unique way. 
Now we define the exact cell in the matrix $A_{k}$ which occupies the given number.

From (1) it follows that if the value of considered number $\mathrm{Z}$ satisfies the inequality $(n-$ 1) $p k !^{\prime}+2 \leq Z<n p k !^{\prime}+2$, then it is the column with the serial number $j k=n$. Therefore, the serial number of the column, in which the considered number $Z$ should be placed must be equal to $j_{k}=$ integer $\left(\frac{Z-2}{p_{k} !}\right)+1$.

On the other hand from (1) we also find that the serial number of search row must be $1 \leq i_{k} \leq$ $i_{k, \max }=p_{k} !^{\prime}$. From (1) it follows that $i_{k}=l_{k}-1$. Therefore, the serial number of the column, in which the given number $Z$ is located, determined by the equation $i_{k}=r_{k}+1$, where $r_{k}$ - the remainder, obtained from dividing $(Z-2)$ on $p_{k} !^{\prime}$, i.e.:

\section{Lemma 1 is proved.}

$$
(Z-2)=r_{k}\left(\bmod p_{k} !^{\prime}\right)
$$

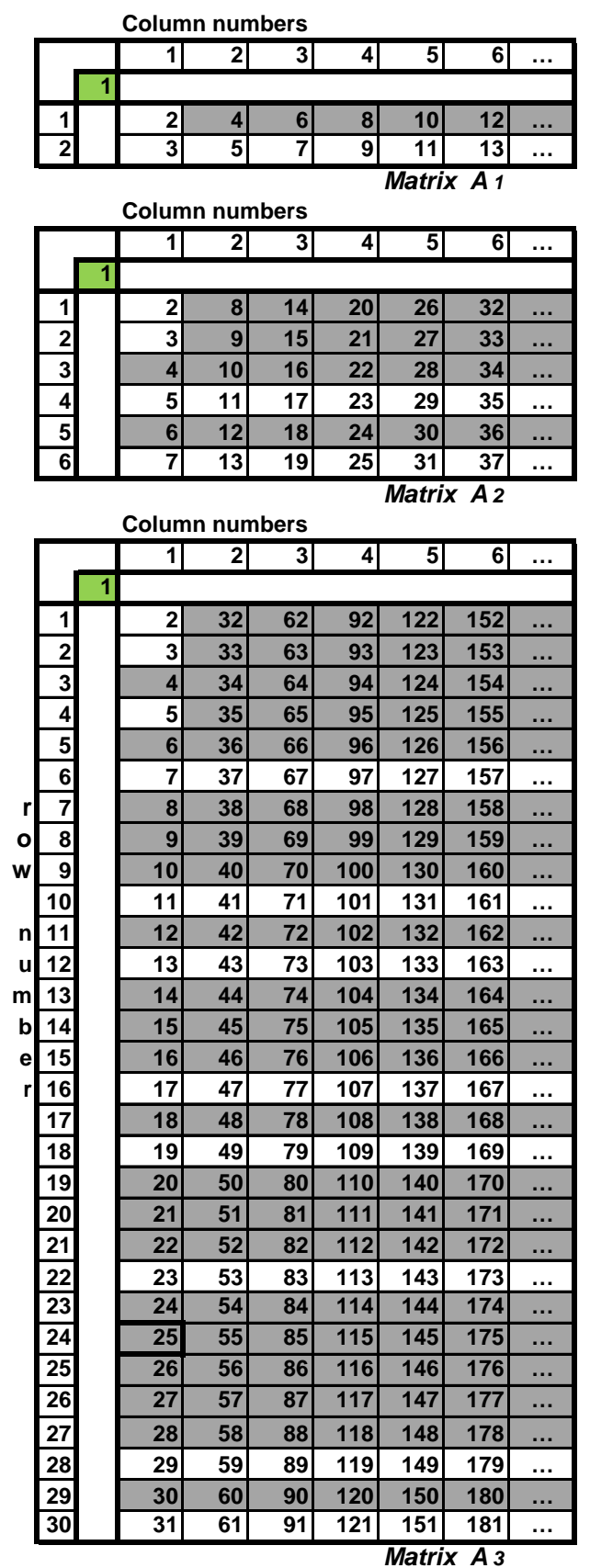
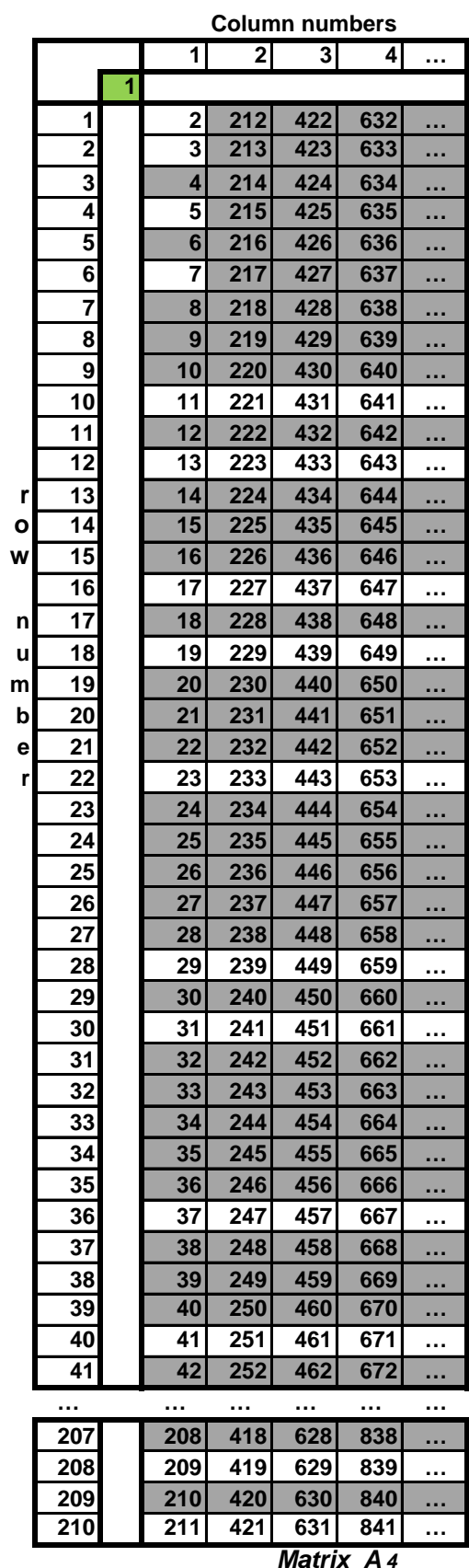

Fig.1. Matrix of prime numbers ${ }^{l}$

\footnotetext{
${ }^{1}$ The number of rows of matrix $A_{4}$ exceeds the paper size, so here its fragment is shown. For this reason, other matrices are not shown in this figure.
} 
Now the comparison of matrices $A_{k}$ and $A_{k+1}$ is considered.

From (1) it follows that each matrix of the matrices family $A_{k}$, where $k=1,2,3, \ldots, \infty$, differs from each other primarily by the number of rows and by the difference of arithmetic progression. For example, if the matrix is considered $A_{k+1}$, then $i_{k+1, \max }=p_{k+1} !$ '. The number of columns in the case $A_{k}$ matrix can be arbitrarily large, up to infinity.

At the same time, an arbitrarily chosen number whether can occupy the same place in the matrices $A_{k}$ and $A_{k+1}$ will be determined?

\section{Lemma 2.}

If the randomly selected number takes the place, located in the first column of the matrix $A_{k}$, it occupies the same place in the matrix $A_{k+1}$, but in other cases it is in the matrices $A_{k}$ and $A_{k+1}$ takes different places.

\section{Proof of lemma 2.}

Consider the number $Z=a\left(k, i_{k}, j_{k}\right)$ from the matrix $A_{k}$ and also consider the this number $Z$ from the matrix $A_{k+1}$, i.e. $Z=a\left(k+1, i_{k+1}, j_{k+1}\right)$. Then

$$
Z=a\left(k+1, i_{k+1}, j_{k+1}\right)=a\left(k, i_{k}, j_{k}\right)
$$

or

$$
\begin{array}{rlr}
a\left(k, i_{k}, j_{k}\right) & =\left(i_{k}+1\right)+p_{k} !^{\prime}\left(j_{k}-1\right) & (3-1) \\
a\left(k+1, i_{k+1}, j_{k+1}\right) & =\left(i_{k+1}+1\right)+p_{k+1} !^{\prime}\left(j_{k+1}-1\right) & (3-2)
\end{array}
$$

Suppose that this number takes the same place in the matrices $A_{k}$ and $A_{k+1}$, i.e. runs equality:

$$
i_{k}=i_{k+1} \quad \text { и } \quad j_{k}=j_{k+1}
$$

From (3-1) and (3-2) we get:

$$
p_{k} !^{\prime}\left(j_{k}-1\right)=p_{k+1} !^{\prime}\left(j_{k+1}-1\right)
$$

From (4) we get that the last equality is possible when performed equality $j_{k}=j_{k+1}=1$. I.e., if the arbitrarily selected number takes place, located in the first column of the matrix $A_{k}$, it occupies the same place in the matrix $A_{k+1}$.

At the same time, as shown above, $i_{k} \leq p_{k} !^{\prime}$. In other cases, it is in the matrices $A_{k}$ and $A_{k+1}$ occupies a different place.

From Fig. 1 clearly shows that the number 2-7, who were in first column $A_{2}$, occupy exactly the same place in the matrix $A_{3}$. The number $2-31$, who were in first column $A_{3}$, occupy exactly the same place in $A_{4}$ matrix.

\section{Lemma 2 is proved.}

Now consider the set of numbers that are in one arbitrary row of the matrix $A_{k}$. Suppose that the serial number of this row is denoted by $n$, i.e. $\boldsymbol{i}_{\boldsymbol{k}}=\boldsymbol{n}$. At the same time $1 \leq n \leq p_{k} !^{\prime}$. Define where each number of given row can be located in case of matrix $A_{k+1}$.

\section{Lemma 3.}

The set of numbers located in one selected row of the matrix $A_{k}$, are redistributed by $p_{k+1}$ rows of the matrix $A_{k+1}$. The sequence of numbers contained in each of these $p_{k+1}$ rows, is an arithmetic progression with a constant $D_{k+1}=p_{k+1} ! '$.

\section{Proof of lemma 3.}

Consider one arbitrary row of $A_{k}$ matrix. Suppose that $n$ - the serial number of given row $(1 \leq n \leq$ $\left.p_{k} !^{\prime}\right)$. From (1) it follows that the sequence of numbers, which are located in any row of the matrix $A_{k}$, is an arithmetic progression, which for this case takes the form:

$$
a\left(k, i_{k}, j_{k}\right)=a\left(k, n, j_{k}\right)=(n+1)+p_{k} !^{\prime}\left(j_{k}-1\right),
$$

where $j_{k}=1,2, \ldots, \infty ; i_{k}=n, 1 \leq n \leq p_{k} !^{\prime}$. 
Define, where each number of progression (5) can be located in the matrix $A_{k+1}$. Initially, from progression (5) select the first $p_{k+1}$ numbers, serial numbers which in the case of the matrix $A_{k}$ are equal to $j_{k}=1,2,3, \ldots, p_{k+1}$. Then from (3) and (5) for case of the matrix $A_{k+1}$ we find that

$$
j_{k+1}=\operatorname{integer}\left(\frac{(n-1)+p_{k} !^{\prime}\left(j_{k}-1\right)}{p_{k+1} !^{\prime}}\right)+1=1,
$$

where $1 \leq j_{k} \leq p_{k+1}$. I.e. from (6-1) it follows that these numbers should be located in the first column of matrix $A_{k+1}$.

On the other hand, from Lemma 1 follows that any number in any matrix is presented in a unique way. This means that the considered above number is, as already indicated above, in one (first) column of matrix $A_{k+1}$, which must be located in different rows. I.e. the numbers in the case of the matrix $A_{k+1}$ should be reallocated for its $p_{k+1}$ rows.

Serial numbers of these rows are determined from (2) and (5):

$$
i_{k+1}=r_{k+1}+1
$$

where $r_{k+1}$ - residue, obtained by dividing the $(n-1)+p_{k} !^{\prime}\left(j_{k}-1\right)$ on $p_{k+1} !^{\prime}$ :

$$
\left[(n-1)+p_{k} !^{\prime}\left(j_{k}-1\right)\right]=r_{k+1}\left(\bmod p_{k+1} !^{\prime}\right), \quad j_{k}=1,2, \ldots, p_{k+1} \quad(6-3)
$$

From (6-2) and (6-3), we find that in this case the sequence numbers of rows, on which the considered numbers are redistributed, should not exceed $p_{k+1} !^{\prime}$, т.e. $i_{k+1}=r_{k+1}+1 \leq p_{k+1} !^{\prime}$.

Now from progression (5) consider $p_{k+1}$ numbers, which follow after the considered above $p_{k+1}$ numbers. Serial numbers of these numbers in case of matrix $A_{k}$ equal to $j_{k}=p_{k+1}+1, p_{k+1}+$ $2, p_{k+1}+3, \ldots, 2 p_{k+1}$. Then from (2) and (5) for the case of the matrix $A_{k+1}$ we find that

$$
j_{k+1}=\operatorname{integer}\left(\frac{(n-1)+p_{k} !^{\prime}\left(j_{k}-1\right)}{p_{k+1} !^{\prime}}\right)+1=2,
$$

where $p_{k+1}<j_{k} \leq 2 p_{k+1}$. I.e. from this equation follows that the number should be located in the second column of the matrix $A_{k+1}$. On the other hand, these numbers, as follows from Lemma 1, should be redistributed by $p_{k+1}$ rows of the matrix $A_{k+1}$. If we substitute the appropriate values $j_{k}$ in (2), we get the same serial numbers of rows, which are obtained under condition $1 \leq j_{k} \leq p_{k+1}$.

Similarly, we find that all numbers of progression (5), which satisfy the condition $m p_{k+1}<j_{k} \leq$ $(m+1) p_{k+1}$, must be located in the same column of the matrix $A_{k+1}$. Serial number of this column is equal to $m+1$. In this case, as in the previous, these numbers must be located indifferent $p_{k+1}$ rows of the matrix $A_{k+1}$. Thus, the serial numbers of these rows will be exactly the same as in the case of (6-2).

As a result, it will be found that the set of numbers of progression (5), i.e. a set of numbers, located in one selected row of the matrix $A_{k}$ redistributed by $p_{k+1}$ rows of the matrix $A_{k+1}$. Serial numbers of these rows are equal $i_{k+1}=n+p_{k} !\left(m_{k+1}-1\right)$, wherem $m_{k+1}=1,2,3, \ldots, p_{k+1}$.

The sequence of numbers in each of these rows is an arithmetic progression with a constant equal to $D_{k+1}=p_{k+1} !^{\prime}$. The value of the first term of an arithmetic progression, located in a separate row of these $p_{k+1}$ rows of the matrix $A_{k+1}$ is equal to $(n+1)+p_{k} !^{\prime}\left(m_{k+1}-1\right)$, where $m_{k+1}=$ $1,2, \ldots, p_{k+1}$.

Consequently, for these rows will be obtained next:

$$
a\left(k+1, i_{k+1}, j_{k+1}\right)=\left[(n+1)+p_{k} !^{\prime}\left(m_{k+1}-1\right)\right]+p_{k+1} !^{\prime}\left(j_{k+1}-1\right),
$$

where $m_{k+1}=1,2, \ldots, p_{k+1}$. Here, at each value $m_{k+1}$ the parameter $j_{k+1}$ has the value from 1 to infinity, i.e. $j_{k+1}=1,2, \ldots, \infty$.

In fact, Figure 1 clearly shows that the numbers located in the same rows, for example in the second row of the matrix $A_{2}$ redistributed by five rows (for the 2-nd, 8-th, 14-th, 20-th and 26-th) of matrix $A_{3}$.

Lemma 3 is proved. 


\section{Lemma 4.}

The set of numbers located in all the rows of the matrix $A_{k}$ are redistributed byp $p_{k+1} !^{\prime}$ rows of the matrix $_{k+1}$.

\section{Lemma proof 4.}

The proof of the lemma is obvious, as the matrix $A_{k}$ has all $p_{k} !^{\prime}$ rows, i.e. $i_{k, \max }=p_{k} !^{\prime}$. On the other hand, from Lemma 3 it follows that the set of numbers located in one selected row of the matrix $A_{k}$ areredistributed by $p_{k+1}$ rows of the matrix $A_{k+1}$.

Consequently, all numbers of this matrix should be reallocated for $p_{k} !^{\prime} p_{k+1}=p_{k+1} !^{\prime}$ rows of the matrix $A_{k+1}$, i.e. total number of rows of the matrix $A_{k+1}$ must be equal to $i_{k+1, \max }=p_{k+1}$ !'.

\section{Lemma 4 is proved.}

It follows that the set of numbers that are in each row of the matrix $A_{k}$ forms an arithmetic progression with a constant $D_{k}=p_{k} !^{\prime}$.

From Dirichlet's theorem for primes in arithmetic progression [4], it follows that if the first term and the difference in progression are not relatively prime, then in this progression will not be any prime number or will be only one prime number. Moreover this prime number is the first term of an arithmetic progression. From Dirichlet's theorem also it follows that if the first term and the difference arithmetic progression are relatively prime, then this progression has an infinite set of primes and composite numbers.

Therefore, in the case of matrix $A_{k}$ set of prime numbers must be located in rows, the first term and the difference arithmetic progressions of which individually are relatively prime. If $D_{k}>2$, then it is obvious that the twin-primes may not be located in the same row. Twins can be found in two adjacent rows, the first term and the difference arithmetic progression of which are relatively prime, and on the other hand the difference between the serial numbers of the two adjacent rows should be equal to 2 . A pair of such adjacent rows is called a couple of rows-twin or rows -twin. For two numbers arranged in different rows, but in the one column of the pairs of twins-rows the equality always takes place $|a(k, i, j)-a(k, i \pm 2, j)|=2$. A pair of such primes are twins. For example, from Figure 1 it is seen that the number (5 and 7), (29 and 31), (41 and 43) and others are twins

If the row has the set of prime and composite numbers, but it is located on the same row at a distance of more than 2, then this row is called single row. Therefore, in single rows cannot be prime numberstwins.

The aim of this work is to determine the total number of primes-twins. Therefore, in the future will be focusing on the pairs of rows-twins.

\section{An Infinite Amount of Prime Numbers-Twins}

As mentioned above, all twin prime are mainly in the pair of rows-twins. If in this case at some point, for example, when considering $A_{k}$ matrix disappear all pairs of rows-twins (i.e. they for some reason have become conventional or single rows), it is obvious that in the following matrices they will not be. In this case, it means that the number of twins should be limited.

Consider the number of rows of $A_{k}$ matrix on which the prime numbers are distributed. In Figure 1 all the rows in which there are only composite numbers (or with only one prime number), for clarity repainted by dark color. Consequently, in each unpainted row there is an infinite set of prime and composite numbers.

Theorem 1. The number of unpainted rows of $A_{k}$ matrix, on which primes are distributed, is determined by the Euler function $\varphi\left(D_{k}\right)$, where $D_{k}=p_{k}$ !'- constant arithmetic progressions of numbers that are in each of these rows.

\section{The proof of theorem 1.}

By definition the Euler $\varphi\left(D_{k}\right)$ function determines the number of positive integers $l_{k}$, not exceeding $D_{k}$ and which are relatively prime ${ }^{2}$ with $D_{k}$, i.e. $1 \leq l_{k} \leq D_{k}$ and $\left(D_{k}, l_{k}\right) \equiv 1$.

\footnotetext{
${ }^{2}$ For our arithmetic progressions the condition $(2 \leq l \leq D+1)$ performing, follows from (1) and fig 1 . If the
} 
On the other hand, from Dirichlet's theorem it follows that if the first term and the difference arithmetic progression are relatively prime, then in this progression there are infinitely many primes.

Consequently, the number of rows of the matrix $A_{k}$, on which distributed an infinite number of prime numbers equal to the value of Euler's function $\varphi\left(D_{k}\right)$ :

$$
n_{k}=\varphi\left(D_{k}\right)=\varphi\left(p_{k} !^{\prime}\right)=\left(p_{1}-1\right)\left(p_{2}-1\right) \ldots\left(p_{k}-1\right)=\left(p_{k}-1\right) !^{\prime} .
$$

\section{Theorem 1 is proved.}

\section{Theorem 2.}

Prime numbers, which are in one unpainted row of the matrix $A_{k-1}$, are redistributed by $p_{k}-1$ unpainted rows of $A_{k}$ matrix.

\section{The proof of theorem 1.}

From Theorem 1 it follows that in the case of the matrix $A_{k-1}$ prime numbers are redistributed by $\varphi \varphi\left(D_{k-1}\right)=\varphi\left(p_{k-1} !^{\prime}\right)=\left(p_{k-1}-1\right) !^{\prime}$ rows. In the case of matrix $A_{k}$ redistribution of primes occur at $\varphi\left(D_{k}\right)=\varphi\left(p_{k} !^{\prime}\right)=\left(p_{k}-1\right) !^{\prime}$ rows. Consequently, if we consider the set of prime numbers that are in one of the unpainted rows of $A_{k-1}$ matrix, then their reallocation in the case of matrix $A_{k}$ occurs by uncolored rows, the number of which is equal to

$$
\frac{\varphi\left(D_{k}\right)}{\varphi\left(D_{k-1}\right)}=\frac{\left(p_{k}-1\right) !^{\prime}}{\left(p_{k-1}-1\right) !^{\prime}}=p_{k}-1
$$

Theorem 2 is proved.

Now consider that all prime numbers taking part in the redistribution mentioned in Theorems 1 and 2 .

\section{Corollary of Theorem 2.}

Consider matrices $A_{k}$. Members of the progressions that are in the rows of this matrix defined by expression:

$$
a\left(k, i_{k}, j_{k}\right)=\left(i_{k}+1\right)+p_{k} !^{\prime}\left(j_{k}-1\right),
$$

where $i_{k}=1,2, \ldots, p_{k} !^{\prime}$ and $j_{k}=1,2, \ldots, \infty$.

From this equation follows that if the first term $\left(i_{k}+1\right)$ and the difference $\left(p_{k} !^{\prime}\right)$ of the progression located in one of these $p_{k} !^{\prime}$ rows, are not mutually prime, then, according to the corollaries of Dirichlet's theorem, there are no prime numbers in this row or only one prime number. I.e. the condition must be fulfilled:

$$
\left(\left(i_{k}+1\right), p_{k} !^{\prime}\right) \not \equiv 1
$$

This condition occurs when $i_{k}+1$ takes the value of the set of composite numbers, which do not exceed $p_{k} ! '$, and in particular the value of the following $k$ prime numbers:

$$
\left(i_{k}+1\right)=p_{1}, p_{2}, p_{3}, \ldots, p_{k}
$$

This means that in the case of the matrix $A_{k}$, the following primes $p_{1}, p_{2}, p_{3}, \ldots, p_{k}$ will not participate in the redistribution of the set of primes by its $\left(p_{k}-1\right) !^{\prime}$ uncolored rows. Similarly, if we consider the matrix $A_{k+1}$, then the following primes $p_{1}, p_{2}, p_{3}, \ldots, p_{k}$ and $p_{k+1}$ will not participate in the redistribution of primes by its $\left(\left(p_{k+1}-1\right)\right.$ !' rows. I.e. in this case the number of prime numbers not participating in redistributions will be 1 more. If we consider the matrix $A_{k-1}$, then the number of primes not participating in the redistribution will be $k-1$, i.e. By 1 less than in the case of matrix $A_{k}$. This happens in any matrix.

number 1 is transferred to the matrix $A_{k}$, then all the members of the last row shift to the first row and the penultimate row becomes the last row. Then the condition is $1 \leq l \leq D$ fulfilled automatically. Therefore, all the conclusions obtained for the conditions $1 \leq l \leq D$ are applicable and are used for our cases. We notice that in this case the first number of this last rows will be equal $l=D=p_{k} !^{\prime}$. Thus it is a row of composite numbers, that is the colored row. 


\section{Theorem 3.}

The number of pairs of twins-rows in the matrix $A_{k}$ increases monotonically with the growth of the serial number $k$ of the matrix, and in each row of any pair of twins-rows there are an infinite number of primes.

\section{Proof of the theorem 3.}

Suppose that there are $m_{k-1}$ pairs of twin rows in this matrix $A_{k-1}$. It follows from Lemma 3 that all the numbers in one of these pairs, in the case of the matrix $A_{k}$, are redistributed by its $p_{k}$ pairs of rows.

Now consider the first row of the selected pair of twins-rows of the matrix $A_{k-1}$. It follows from Theorem 2 that all prime numbers in this row are redistributed over the $p_{k}-1$ rows of the matrix $A_{k}$. This means that from the analyzed $p_{k}$ pairs of rows of the matrix $A_{k}$, one pair ceases to be a pair of twins-rows.

If we consider the redistribution of prime numbers in the second row of the pair of twins-rows of the matrix $A_{k-1}$, we similarly get that in this case one more pair from $p_{k}$ pairs of rows of the matrix $A_{k}$ ceases to be a pair of twins-rows.

As a result, we obtain that all primes in an arbitrary pair of twin rows of matrix $A_{k-1}$, in the case of matrix $A_{k}$, are redistributed by its $p_{k}-2$ pairs of twins-rows. Note that the first number and the difference of the progression, consisting of the numbers located in each of these rows, are relatively prime.

From the above, we also note that as a result from the analyzed $p_{k}$ pairs of rows of the matrix $A_{k}$, two pairs of rows cease to be a pair of twins-rows, i.e. in the matrix present 2 ordinary rows and 1 pair of 2 single rows.

If we take into account that there are $m_{k-1}$ pairs of twins-rows in the matrix $A_{k-1}$, then the total number of pairs of twin-rows in the matrix $A_{k}$ will be:

$$
m_{k}=m_{k-1}\left(p_{k}-2\right)
$$

Moreover, in the matrix $A_{k}$, the number of pairs of single rows generated from these $m_{k-1}$ pairs of twins-rows of the matrix $A_{k-1}$ will be equal to

$$
m_{k}^{\prime}=m_{k-1}
$$

As shown above, there is only one pair of twins-rows in the matrix $A_{2}$. With this in mind, it can be established that $m_{k}$ the number of pairs of twins-rows in the matrix $A_{k}$ is determined by the following expression:

$$
m_{k}=\left(p_{2}-2\right)\left(p_{3}-2\right) * \ldots *\left(p_{k}-2\right)=\left(p_{k}-2\right) !^{\prime}, \text { where } k \geq 2
$$

It follows from (9) that as the serial number $k$ of the matrix $A_{k}$ increases, the number of pairs of twins-rows in it grows monotonically. On the other hand, the sequence of numbers in each row of $m_{k}$ pairs of twins-rows is an arithmetic progression. Moreover, as shown above, the first term and the difference of each of these progressions are mutually simple (prime). In view of this, it follows from the Dirichlet theorem that every row of any pair of twins-rows has an infinite number of primes.

\section{Theorem 3 is proved.}

It follows from (9) that $\lim _{k \rightarrow \infty} m_{k}=\infty$, i.e., at $k \rightarrow \infty$ the number of pairs of twins-rows of the matrix $A_{k}$ tends to infinity. If we assume that in each pair of twin rows of $A_{k}$ matrix there is at least one pair of primes-twins, then one can say unequivocally that the number of twins is infinite. And this is possible when the prime numbers in each row of any pair of twin rows of the matrix $A_{k}$ are located more closely and densely than in the case of the matrix $A_{k-1}$.

In this case, from an infinite set of prime numbers located in each of the two rows of any pair of twins-rows, at least 2 prime numbers will necessarily be in the same column and thus they will be twins.

Is it so? Let us analyze this with the help of the following theorems.

First we consider the set of prime and composite numbers contained in the matrix $A_{k}$ and not 
exceeding $x$ such that $x \gg D_{k}=p_{k} !^{\prime}$. This means that in the future we will consider fragments of matrices $A_{k}$. In this case, to each matrix $A_{k}$ will correspond its fragment $B_{k}$, consisting of $i_{k, \max }$ rows and $j_{k, \max }$ columns:

$$
\begin{gathered}
i_{k, \max }=D_{k}=p_{k} !^{\prime} \\
j_{k, \max }=\frac{x}{D_{k}}=\frac{x}{p_{k} !^{\prime}}=m_{k} \gg 1
\end{gathered}
$$

It follows that if $m_{k}$ is an integer, then the considered fragment $B_{k}$ is a two-dimensional full-scale matrix. And if $m_{k}$ is not an integer, then the last column of the considered fragment $B_{k}$ will be short, than its other columns. Therefore, in the sequel, $x$ will be chosen so that $m_{k}$ is always an integer.

Note that if all considered numbers do not exceed $x$, then as the serial of considered fragment of the matrix increases, the number of its rows increases monotonically to $i_{k, \max }=D_{k}=p_{k} !^{\prime}$, and the number of columns decreases monotonically to $j_{k, \max }=\frac{x}{p_{k} ! !^{\prime}}=m_{k}$. I. e. fragments of matrices $B_{1}, B_{2}, \ldots, B_{k}$ differ from each other, first of all, by the number of rows and columns.

In this case we have the following lemma.

\section{Lemma 5.}

If the arbitrary number $Z$, not exceeding $x, Z \in B_{k}$, then $Z \in B_{w}$, where $n=0,1,2, \ldots, k$.

\section{Proof of the lemma 5.}

Let $Z$ be a arbitrary number not exceeding $x$.

We imagine a fragment of random matrix $B_{k}$ as plurality of cells arranged in all rows and columns of the matrix (figure 1). Only one number can be located in every cell, and the cell must correspond to a certain pair of coordinates $\left(i_{k}\right.$ and $\left.j_{k}\right)$. Then the total number of cells, i.e. the total quantity of numbers in consideration fragment, is equal

$$
i_{k, \max } * j_{k, \max }=D_{k} * m_{k}=p_{k} !^{\prime} * \frac{x}{p_{k} !^{\prime}}=x
$$

Therefore, total quantity of numbers that contained in a fragment of the matrix $B_{k}$, independent of its sequence number $k$.This means that the quantity of prime and composite numbers, the total quantity is $x$, is not dependent on the ordinal number of the matrix fragment $B_{k}$, where they located. Explanation: not one of these composite number, depending on the ordinal number of the matrix doesn't become a prime number, and conversely. The number in itself is a prime number, and not depending on anything, as a composite number. This means, if a number of $\mathrm{Z}$ is not more than $x$, then it must be in all fragments of the matrix $B_{n}$, where $n=0,1,2, \ldots, k$. From lemma 1 followed that the number in the current fragment have the only place. Its coordinates are defined by using the equality (2).

\section{Lemma 5 is proved.}

Now we consider the new parameters. Let $\pi_{k}, n_{k}$ and $N_{k}$ is a quantity of prime, composite and all the numbers existing in one of the uncolored rows fragments of the matrix $B_{k}$ and not exceeding $x$ such that $x \gg D_{k}=p_{k} !^{\prime}$. Let $\pi_{0}, n_{0}$ иN $N_{0}$ is a quantity of prime, composite and all the numbers containing in an arithmetic progression, consisting of a natural numbers from 1 to $x$.

Define the ratio $\frac{v_{k}}{v_{0}}$, where $v_{0}=\frac{\pi_{0}}{n_{0}}$ and $v_{k}=\frac{\pi_{k}}{n_{k}}$ - are parameters that show the number of prime numbers per one composite number.

\section{Theorem 4.}

For prime numbers that are in any uncolored rows fragments of the matrix $B_{k}$, hold true the following formulas:

$$
\pi_{k}=\frac{\pi_{0}-k}{\varphi\left(p_{k} !^{\prime}\right)}
$$




$$
\ln v_{k}=\ln v_{0}+\left(\sum_{i=1}^{k} \frac{1}{p_{i}}+\sum_{i=1}^{k} \sum_{m=2}^{\infty} \frac{1}{m * p_{i}^{m}}\right)+o(1)
$$

\section{Proof of the theorem 4.}

Now, consider fragments of the matrix $B_{k-1}$ and $B_{k}$. From theorem 1 followed that prime numbers, which were distributed on $\varphi\left(D_{k-1}\right)$ rows of the fragment $B_{k-1}$, in case the fragment $B_{k}$, redistributed on its $\varphi\left(D_{k}\right)$ rows. From conclusion of the Theorem 2 and Lemma 5 we find that this redistribution takes place without the participation of one prime $p_{k}$. For this reason have the space the following equality:

$$
\begin{aligned}
& \pi_{1} \varphi\left(D_{1}\right)=\pi_{0} \varphi\left(D_{0}\right)-1 \\
& \pi_{2} \varphi\left(D_{2}\right)=\pi_{1} \varphi\left(D_{1}\right)-1=\pi_{0} \varphi\left(D_{0}\right)-2 \\
& \pi_{3} \varphi\left(D_{3}\right)=\pi_{2} \varphi\left(D_{2}\right)-1=\pi_{0} \varphi\left(D_{0}\right)-3 \\
& \pi_{k} \varphi\left(D_{k}\right)=\pi_{k-1} \varphi\left(D_{k-1}\right)-1=\pi_{0} \varphi\left(D_{0}\right)-k
\end{aligned}
$$

Where $\pi_{0}$ - is the quantity of prime numbers containing in an arithmetic progression, consisting of a natural numbers from 1 to $x$. We know that $\varphi\left(D_{0}\right)=\varphi(1)=1$.

From the last equality follows that

$$
\pi_{k}=\frac{\pi_{0}-k}{\varphi\left(p_{k} !^{\prime}\right)}
$$

where $k$ - is the ordinal number of the matrix.

We define $N$. All numbers in the case of matrix fragment $B_{k-1}$ containing in its $p_{k-1}$ !' rows, evenly redistributed by $p_{k}$ !' rows of the matrix fragment $B_{k}$, follows from Lemma 4 . Therefore, all numbers in an arithmetic progression consisting of natural numbers from 1 to $x$, redistributed by $p_{k}$ !' rows of the matrix fragment $B_{k}$, i.e.:

$$
N_{k} p_{k} !^{\prime}=N_{0}
$$

where $N_{0}$ - is the number of all (prime and composite) numbers, that contained in an arithmetic progression consisting of a natural numbers from 1 to $x$, i.e. $N_{0}=x$

We obtain from the last equality:

$$
N_{k}=\frac{N_{0}}{p_{k} !^{\prime}}=\frac{x}{p_{k} !^{\prime}}
$$

The quantity of composite numbers not exceeding $x$ and containing in a separate row of the selected uncolored fragment $B_{k}$ of matrix determined, we define from (10), (11)

$$
\begin{gathered}
n_{k}=N_{k}-\pi_{k}=\frac{x}{p_{k} !^{\prime}}\left(1-\varepsilon_{1}\right), \\
\varepsilon_{1}=\frac{\left(\pi_{0}-k\right) p_{k} !^{\prime}}{x \varphi\left(p_{k} !^{\prime}\right.}
\end{gathered}
$$

where $\varepsilon_{1}$ - is a small quantity

We define the ratio of prime numbers to the composite number, containing in uncolored rows of the matrix fragment $B_{k}$ from (10), (12):

$$
\begin{gathered}
v_{k}=\frac{\pi_{k}}{n_{k}}=\frac{\pi_{0}\left(1-\frac{k}{\pi_{0}}\right) p_{k} !^{\prime}}{x \varphi\left(p_{k} !^{\prime}\right)\left(1-\varepsilon_{1}\right)}=\frac{\pi_{0} p_{k} !^{\prime}}{x \varphi\left(p_{k} !^{\prime}\right)}\left(1+\varepsilon_{1}\right)\left(1-\varepsilon_{2}\right), \\
\varepsilon_{2}=\frac{k}{\pi_{0}}-\text { is a small quantity }
\end{gathered}
$$

Similarly for the matrix fragment $B_{k}$ get: 


$$
\begin{gathered}
v_{k-1}=\frac{\pi_{k-1}}{n_{k-1}}=\frac{\pi_{0} p_{k-1} !^{\prime}}{x \varphi\left(p_{k-1} !^{\prime}\right)}\left(1+\varepsilon_{1}\right)\left(1-\varepsilon_{2}^{\prime}\right), \\
\varepsilon_{2}^{\prime}=\frac{k-1}{\pi_{0}}-\text { is a small quantity }
\end{gathered}
$$

If consider sequence of natural numbers in the range from 1 to $x$, then $x=n_{0}+\pi_{0}$, therefore from (13) follows, that:

$$
\begin{gathered}
v_{k}=v_{0} \frac{p_{k} !^{\prime}}{\varphi\left(p_{k} !^{\prime}\right)}\left(1+\varepsilon_{1}\right)\left(1-\varepsilon_{2}\right)\left(1-\varepsilon_{3}\right), \\
\varepsilon_{3}=\frac{\pi_{0}}{n_{0}}-\text { is a small quantity }
\end{gathered}
$$

or

$$
v_{k}=v_{0} \frac{p_{k} !^{\prime}}{\left(p_{k}-1\right) !^{\prime}}\left(1+\varepsilon_{1}\right)\left(1-\varepsilon_{2}\right)\left(1-\varepsilon_{3}\right),
$$

If using (12-2), (13-2) and (15-2), then multiplication $\left(1+\varepsilon_{1}\right)\left(1-\varepsilon_{2}\right)\left(1-\varepsilon_{3}\right)$ is a limited quantity.

From (13-1), (14-1) and (15-1) followed, that a prime numbers in any row uncolored fragment of the matrix $B_{k}$ placed more closely than in the case of the previous fragments of the matrix $B_{k-1}$. From (15) also followed that if the ordinal number $k$ of a fragment of the matrix $B_{k}$ increases, then the ratio $\frac{v_{k}}{v_{0}}$ grows continuously in any of its uncolored row.

Choose $x$ indeed very large, let be $x \rightarrow \infty$. Then this $k$ also can be very large, let be $k \rightarrow \infty$. Then after simple transformation from (16) get that:

$$
\ln v_{k}=\ln v_{0}+\left(\sum_{i=1}^{k} \frac{1}{p_{i}}+\sum_{i=1}^{k} \sum_{m=2}^{\infty} \frac{1}{m * p_{i}^{m}}\right)+o(1)
$$

An infinite sum consisting of reciprocals primes diverges, as shown by Euler. Here it is: $\sum_{i=1}^{\infty} \frac{1}{p_{i}}=\infty$.

Therefore, if ordinal number of a fragment of the matrix $B_{k}$ increases, then the parameter $v_{k}$ and $\frac{v_{k}}{v_{0}}$ growing constantly.

\section{Theorem 4 is proved.}

It should be noted that the execution of theorem 4, in particular (16), perhaps then when in any unpainted rows of fragment $B_{k}$ the primes placed more tightly than in case of previous fragments $B_{i}$, where $i<k$. This means that in each pair of rows of twins will undoubtedly twins.

Now consider the problem, posed before this work.

\section{Theorem 5.}

\section{The hypothesis of an infinite quantity of prime twins is true.}

\section{Proof of the theorem 5.}

We consider a couple of rows of the twins in a fragment of the matrix $B_{k}$ when $k$ is large. Let, this pair of rows contains a lot of prime and composite numbers, not exceeding $x$.

Assume, there is no pair of twins in this pair of rows. Two prime of different rows can't contains in one column of the pair of twin rows. It is possible when $\frac{v_{k}}{v_{0}}<1$.

This conclusion contradicts to Theorem 4, in particular (16). Therefore, there are twins in each pair of twin rows.

If ordinal number of a fragment of the matrix $A_{k}$ increases, then the quantity of pair of rows-twins growing constantly. This dependence (conclusion) follows from theorem 3 : 


$$
\lim _{k \rightarrow \infty} m_{k}=\lim _{k \rightarrow \infty}\left(p_{k}-2\right) !^{\prime}=\infty
$$

So, there are twins in each of these pairs of rows-twins. This means that the number of twins infinitely.

\section{Theorem 5 is proved.}

\section{CONClusion}

In this paper, to study the properties of prime twin numbers, the notion of a matrix of primes is introduced. Then in the paper we prove a number of lemmas and theorems with the help of which and the Dirichlet and Euler theorems are proposed to prove the infinity of the number of prime twins.

\section{REFERENCES}

[1] Goldston D. A., Pintz J. \& Y1ldırım C. Y., Ann. Math. 170, (2009), p.819-862.

[2] Baibekov S.N.,About the Infinite Number of Twin Primes, Search. 1, (2016), p.203-213.

[3] Baibekov S.N., Durmagambetov A.A., Infinite Number of Twin Primes. Advances in Pure Mathematics.6, (2016), p.954-971.

[4] Postnikov M.M. The Introduction to the theory of algebraic numbers. M.: Science, 1986.

\section{AUTHOR's BIOGRAPHY}

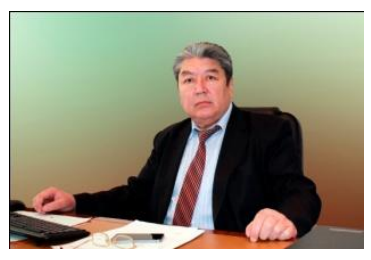

\section{Baibekov S.N.}

Nationality - Republic of Kazakhstan

Education - Physical faculty of Leningrad State University

Academic Degree - Doctor of technical science

Academic Title- Professor

Author of more than 130 scientific articles and about 10 monographs, textbooks and manuals Academic interests - Mathematics (number theory), Physics, IT-Technology and others.

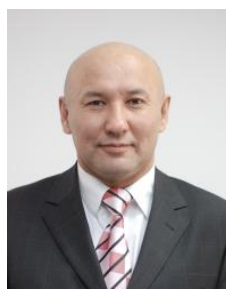

Durmagambetov A.A., Specialist, senior scientific employee, managinging laboratory of Applied Mathematics.

Management of program-targeted designed by Ministry of Education RK "Development of complex scientific and technical solutions, theoretical and experimental research prototype of robotic systems"

Citation: Baibekov S. N., "Proof of an Infinite Number of Primes-Twins", International Journal of Scientific and Innovative Mathematical Researc, vol. 5, no. 4, p. 12, 2017., http://dx.doi.org/10.20431/23473142.0504002

Copyright: () 2017 Authors. This is an open-access article distributed under the terms of the Creative Commons Attribution License, which permits unrestricted use, distribution, and reproduction in any medium, provided the original author and source are credited. 